\title{
Fungal mycotoxins and natural antioxidants: Two sides of the same coin and significance in food safety
}

\author{
Darwish AMG* \\ Food Technology Department, Arid Lands Cultivation Research Institute (ALCRI), City of Scientific Research and \\ Technological Applications (SRTA-City), Alexandria, Egypt.
}

Darwish AMG 2019 - Fungal mycotoxins and natural antioxidants: Two sides of the same coin and significance in food safety. Microbial Biosystems 4(1), 1-16.

\begin{abstract}
Fungi have a long and intimate connection with humankind, particularly at the chemical level as a source of both harmful and beneficial compounds. Their worldwide occurrence in various food and feeds poses a major health threatening risk for human and animal and, as a consequence, mycotoxins represent an economic burden. Fortunately, not all fungal secondary metabolites are toxic to humankind such as; antibiotics, phytotoxines, enzymes and antioxidants, which represent the other side of the coin. Endophytes are now considered as an important component of biodiversity, and act as reservoirs of novel bioactive secondary metabolites, such as alkaloids, flavanoids, phenols, phenolic acids, quinones, steroids, saponins, tannins, terpenoids, isocoumarin derivatives, peptides and enzymes, beside their ability to produce the same or similar kind of compounds of their host plants. Today's world challenge is ensuring the safety of food, which has been a major focus of international and national action over the last years. Legislative limits for a range of mycotoxins continue to develop worldwide, due to being carcinogenic to animals and humans, resulting in an increased number of official controls monitoring in the food supply chain deriving from national food safety plans and for food trade purposes. This review brings about the harmful (mycotoxins) and beneficial (antioxidants, antibiotics, enzymes) fungal metabolites in industrial and pharmaceutical applications. It also provides a perspective about mechanism of how can endophytic fungi metabolites play a reverse role as one of the main strategies of mycotoxin deactivation and prevention representing the other side of the coin.
\end{abstract}

Key words - Fungal metabolites, Fungi in cheese, Hazard assessment, Mycotoxins eliminating strategies, Regulations.

\section{Introduction}

Fungi can influence nearly every aspect of ecosystem function; they can decompose organic material to obtain energy and nutrients releasing carbon dioxide as a by-product. On the other hand, they can also produce their own organic compounds by releasing extracellular enzymes. Thus, their activities can have large-scale consequences for global biogeochemical cycles (Treseder et al. 2015).

Food safety is threatened by numerous pathogens and toxins, including mycotoxins, which are associated to a variety of acute and chronic foodborne diseases. The term mycotoxin is derived from the Greek word 'mycos' meaning fungus (mold), and the Latin word 'toxicum', which means poison. Mycotoxins are toxic fungal secondary metabolites of enormous chemical permits unrestricted use, distribution, and reproduction in any medium, provided the original author and source are credited. 
diversity. Their economic costs are impossible to estimate accurately, but the United States Food and Drug Administration (FDA) in 2007 evaluated the potential economic losses in USA due to mycotoxin contaminations average $\$ 932$ million per year and that $25 \%$ of the world's crops are affected by mycotoxins each year, with annual losses of around 1 billion metric tons of food and food products. From an economic and public health standpoint, the foodborne mycotoxins that are considered as being relevant are aflatoxins (AFs), fumonisins (FBs), certain trichothecene mycotoxins (including deoxynivalenol (DON) and T-2 and HT-2 toxins), ochratoxin A (OTA), patulin (PAT) and zearalenone (ZEA) (Assunção et al. 2016). From the perspective of consumer health protection, it is essential that comprehensive assessment of human and animal health risks related to dietary intake of mycotoxins covers all forms of mycotoxins potentially causing adverse effects in humans and/or animals. In recent years, it has become increasingly evident that matrix-associated mycotoxins as well as chemical and biological modifications of the parent mycotoxin collectively defined as modified mycotoxins may co-occur in addition to the corresponding free or parent compound and may contribute to overall mycotoxin exposure (EFSA 2018).

Since Freeman in 1904, made his four papers on endophytes that were published in 1898; several discoveries prompted a series of studies in which similar asymptomatic endophytes were recorded in a wide range of grasses between 1930-1990. Reports are also available in between 1990-2000 on European endophytes until significant contributions to knowledge of endophytes have been published by several authors from 2000-2012 (Padhi et al. 2013). Diverse endophytic fungi reside in plants, representing a rich resource of bioactive natural products with potential for exploitation in pharmaceutical and agricultural field. Their antioxidant metabolites can play an adverse role against oxidative damage caused by mycotoxins which considered being the main mechanism leading to the subsequent hepatotoxicity by promoting lipid peroxidation linked with liver cell injury. The induction of oxidative stress is commonly related to an imbalance between the oxidants and the antioxidant systems (Yilmaz et al. 2018).

The European Union (EU) has been working for almost two decades on the harmonization of my co-toxin standards for cereals and foods, based on international toxicological analysis and established regulations including limits for the commodities and methods of analysis and sampling (Zadeike et al. 2018). Several national and international organizations and agencies have special committees and commissions that set recommended guidelines, develop standardized assay protocols, and maintain up- to-date information on regulatory statutes. There are also several mycotoxin associations. The web sites for these commissions and organizations are excellent sources for the latest information. See, for example, the Council for Agricultural Science and Technology (www.cast-science.org), the Mycotoxicology Newsletter (www.mycotoxicology.org), the Society for Mycotoxin Research (www.mycotoxin.de), the American Oil Chemists Society Technical Committee on Mycotoxins (www .aocs.org), the Food and Agriculture Organization of the United Nations (www.fao.org), the International Union for Pure and Applied Chemistry Section on Mycotoxins and Phycotoxins (www.iupac.org), the Japanese Association of Mycotoxicology (www.chujou.ac.jp/myco/index.html), and the U.S. Food and Drug Administration Committee on Additives and Contaminants (www.fda.gov) (Bennett et al. 2013).

This review brings about the rise and role of harmful (mycotoxins) and beneficial (antioxidants, antibiotics, enzymes) fungal metabolites in industrial and pharmaceutical applications. It also provides a perspective about mechanism of how can endophytic fungi metabolites contribute to play a reverse role as one of the main strategies of mycotoxin deactivation and prevention representing the other side of the coin. Finally, it refers to mycotoxins hazard assessment and regulations stated by worldwide organizations aiming to guarantee public health and food safety. 


\section{Fungal harmful/beneficial metabolites historical interest and discovery}

The discovery that fungi were the source of harm was brought to light immediately after an outbreak of a disease of turkeys of unknown etiology in England in 1960. The disease was called Turkey " $X$ " disease and was attributed to a toxic groundnut meal imported from Brazil which was eventually elucidated that a species of Aspergillus flavus, was responsible for the hepatotoxic products were called aflatoxins, found as components in the toxic groundnut meal. Subsequent, the carcinogenic and immunosuppressive material, aflatoxins caused concern for their occurrence in human foods (Richard 2008).

Imhotep, the real Father of Medicine who was an ancient Egyptian practitioner, used moldy bread to treat infections of the face and wounds. That affected the recent folk medicine which has documented some other examples of the use of molds on infections. For example, moldy jam and moldy bread were widely used in folk- based therapy in Quebec (Canada), Devon (UK), and Kansas (USA) and poultices made from moldy chewed barley and apple have long been used in Asia to cure surface wounds (Alnaimat et al. 2015).

The discovery of the first broad-spectrum antibiotic, penicillin, in 1928, by the Scottish Alexander Fleming from a fungus named Penicillium rubrum, played a crucial role in the treatment and prevention of infections which considered the 'wonder drug' of World War II, opened a new era in the treatment of infectious diseases, described as the "golden age" of antibiotic research (1940-1962) (Penesyan et al. 2015). In 1945, the Italian Giuseppe Brotzu was able to isolate the cephalosporin producer, a filamentous fungus Cephalosporium acremonium extracts (nowadays this fungus is known as Acremonium chrysogenum) cultured from the sea near a Sardinian sewage outfall. He discovered that it produced an effective substance against gram-negative bacteria (including Salmonella typhi), when noticed no outbreaks of thyphoid fever cases related to bathing in "Su Siccu" Bay, precisely at the site where the city sewer system drained into the sea. Later, he was proposed for the Nobel Prize for his discovery of the Acremonium fungus, the cephalosporin producer. His further studies on Acremonium, at the William Dunn School of Pathology in Oxford, indicated that this fungus could produce at least five different antibiotics, at the beginning was cephalosporin $\mathrm{P}$, then followed investigations yielded cephalosporins C and N ( Mandell et al. 1992; Muñiz et al. 2007). The third clinically important antibiotic produced by fungi, after penicillin and cephalosporin was the steroid fusidic acid. This compound is derived from the fungus Fusidium coccineum, and was developed in the early 1960s by the Danish drug company Leo Pharma. It was introduced clinically for the treatment of Gram positive bacterial infections (Langdon et al. 2017)

The interest in Chaetomium enzymes almost has begun when one of the early researchers developed a method for testing the effectiveness of mildew proofing agents on cotton fabrics in which the fungus, Chaetomium globosum, is used as the test organism. As it was found as one of the organisms responsible for the loss of breaking strength of fabrics (Thom et al. 1934). After three decades, the interest switched to studying of the cellulolytic system in more details in the thermophilic compost fungus Chaetomium thermophile var. dissitum (Eriksen et al. 1977) and the isolation, taxonomy and growth rate of the genus Chaetomium Kunze ex Fr. as a wheat straw decomposer for mushroom growth (Chahal et al. 1976). Later, stored useful books which were noticed to have moldy appearances were analyzed for isolation of cellulolytic fungi. Several fungi were isolated and the genus Chaetomium was the most dominant fungus (Yadav et al. 2015).

\section{Role of fungal metabolites in cheese production}

Fortunately, not all toxic compounds produced by fungi are called mycotoxins. The target and the concentration of the metabolite are both important. Fungal products that are mainly toxic to bacteria (such as penicillin) are usually called antibiotics. Fungal products that are toxic to plants are called phytotoxins, while other low-molecular weight fungal metabolites such as 
ethanol that are toxic only in high concentrations are not considered mycotoxins. On the other hand, not all metabolites are toxic such as antioxidants and enzymes that gave great importance to fungi in industrial applications.

Some contaminating fungal species growing on cheese include Penicillium, Aspergillus, Cladosporium, Geotrichum, Mucor, and Trichoderma have the potential to produce undesirable metabolites such as mycotoxins (Hymery et al. 2014). For some cheeses, molds are intentionally added. Different types of fungi ripened cheeses are manufactured and consumed worldwide, displaying specific organoleptic properties (Figuer 1). There are two main types of these cheeses; the blue-veined cheeses, as their name, are those in which blue-green mold can be seen growing throughout their interior, and surface mold-ripened cheeses which is characterized by a white mold growth on the surface and a soft texture when properly aged. Penicillium roqueforti is used as a starter culture for blue-veined cheeses, being responsible for their flavor and color. These features may be due to the different manufacturing methods and/or to the specific $P$. roqueforti strains used. Cheese-making is an ancient process that has led to more than 1000 varieties of cheese known to date. Within the huge cheese diversity, blue-veined cheeses are manufactured from different milks and consumed in numerous countries. The best-known blue cheeses worldwide are, in the order of their first recorded date in the literature, Italian Gorgonzola (879), French Roquefort (1070), English Stilton (1785) and Danish Danablu (1870s). The presence and growth of the mold $P$. roqueforti under harsh conditions such as low temperatures, high carbon dioxide concentrations and/or its resistance to sodium chloride and organic acids, largely contribute to the typical aspect and flavor of blue cheeses. Taxonomically, $P$. roqueforti, genus Penicillium, subgenus Penicillium and species roqueforti, is currently recognized as a single species, although substantial morphological differences has led to numerous distinct “technological" species names such as $P$. glaucum, $P$. stilton, $P$. gorgonzolae or $P$. aromaticum ( Pitt et al. 2009; Gillot et al. 2015).

Penicillium camemberti and Geotrichum candidum are the main fungal microbiota in Camembert-type surface mold-ripened cheese. These species are major contributors to the texture and flavor of typical bloomy rind cheeses. Biochemical studies showed that $P$. camemberti is responsible for the white and bloomy aspect of the rind, and produces enzymes such as lipases that hydrolyze triglycerides into di- and mono-glycerides, free fatty acids (FA) and glycerol in addition to proteases that involve in lipolysis and proteolysis activities, while $G$. candidum reduces bitterness, enhances sulphur flavors through amino acid catabolism and has an impact on rind texture, firmness and thickness (Lessard et al. 2014). 

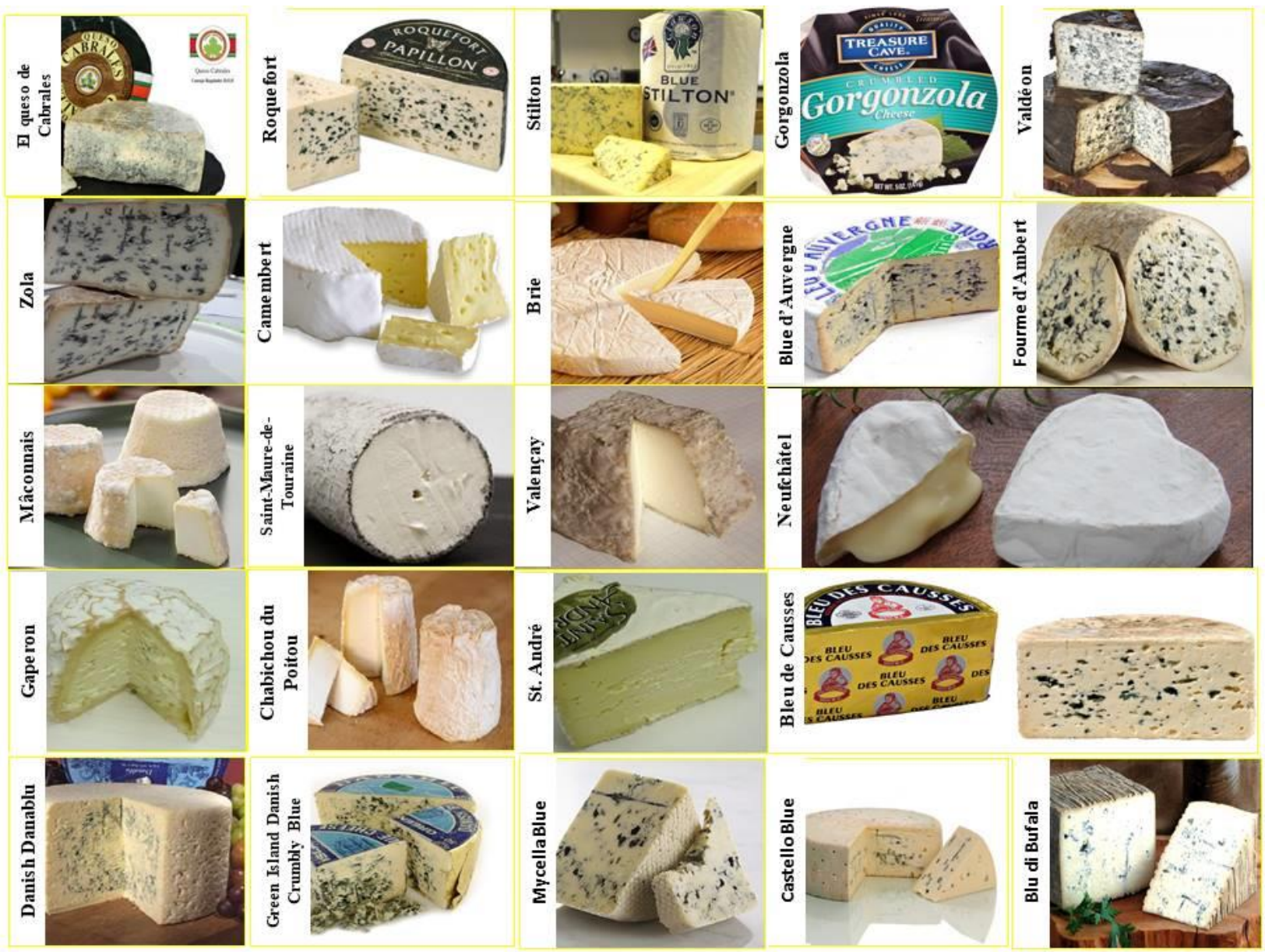

Fig 1 - Types of fungi ripened cheeses manufactured worldwide.

\section{Mining fungi for pharmaceuticals}

Mining fungi for medicine and pharmaceutical continued growing. The bioactivity of fungal metabolites has been known for at least 12 centuries and has led to the development of the most important drugs of the 21 st century. Shiitake mushrooms, contain lentinan, a polysaccharide shown to be effective in enhancing the immune system, kill microbes and viruses. Compounds from shiitakes have also been shown to lower cholesterol. Ergotamine is a vasoconstricting ergot alkaloid from Claviceps purpurea, is used to treat migraine type headaches. It was first isolated by Arthur Stoll at Sandoz (now Novartis) in 1918. Penicillium stoloniferum metabolites with immunosuppressive activity including mycophenolic acid that inhibits purine biosynthesis and effects $\mathrm{B}$ and $\mathrm{T}$ lymphocyte proliferation and was approved by the FDA in 2000. Statins are metabolites of Penicillium compactum and Aspergillus terreus work as cholesterol biosynthesis inhibitors and subsequently affect hydroxymethylglutaryl coenzyme A reductase (HMG-CoA reductase) (Langdon et al. 2017).

Fungi are great producers of enzymes and have contributed enormously to enable and facilitate industrial processes. From food to pharmaceutical products and chemical goods, these enzymes have proven their importance in our everyday lives. Replacement of native lactase through the use of exogenous enzymes, derived from yeast or fungi, may be considered a reliable therapeutic option. Exogenous lactase can be administered with milk, or as capsules/tablets before eating dairy products for lactose intolerance patients (Ianiro et al. 2016). Fungal proteases are used for hydrolyzing protein and other components and have attracted the attention of environmental biotechnologists because fungi can grow on low cost substrates and secrete large amount of enzymes into culture medium which could ease downstream processing (Souza et al. 2015). Fungal enzymes are high-molecular-weight proteins that are catalysts. The most widely 
used enzymes of occupational importance are derived from the genus Aspergillus and include $\alpha$ amylase, xylanase, and cellulase. Other enzymes are also utilized from rhizosphere fungal species belonging to the genera Rhizopus and Humicola (Green et al. 2011). Lipases (triacylglycerol acylhydrolase, EC 3.1.1.3) are part of the family of hydrolases that act on carboxylic ester bonds. The physiologic role of lipases is to hydrolyze triglycerides into diglycerides, monoglycerides, fatty acids, and glycerol. They are very useful in such pharmaceuticals such as anticholesterolemics, anti inflammatories, and thrombolytics, dairy (hydrolysis of milk, fat) and medical (diagnostic tool in blood triglyceride assay) (Suseela and Anupama 2014; Mehta et al. 2017).

\section{Other fungal metabolites applications}

The genus Chaetomium is a rich source of novel and bioactive secondary metabolites of great importance. To date, a variety of more than 200 secondary metabolites belonging to diverse structural types have been discovered. Fungal enzymes are used in food, beverages, confectionaries, textiles, and leather industries to simplify the processing of raw materials. They are often more stable than enzymes derived from other sources (Abdel-Azeem et al. 2016).

Lactases are $\beta$-galactosidases, enzymes that catalyze the hydrolysis of lactose into galactose and glucose which is highly important in the dairy industry and a relief for people who suffer from lactose intolerance. Aspergillus oryzae which has fundamental importance as generally recognized as safe (GRAS) from the Food and Drug Administration (FDA), has been approved in producing the enzyme in sufficient amounts, being commercially available and used in the milk industry (Abdel-Azeem et al. 2019a).

Novel fungi that have capability to synthesize unique polysaccharide with antioxidant properties became the scientists' target. Carbohydrate antioxidants are expected to have better applicability as they are easily isolated, purified, water soluble and have less chances of toxicity towards cell. Fungal EPSs have several applications in the food, feed, cosmetic, medicine, and pharmaceutical industries. The activities of fungal carbohydrate compounds are dependent on different content and arrangements during polymerization of its building unit, monosaccharides. Their composition varies from pure sugars to sugars combined with a second unit such as protein, phosphate, sulfate, or amine. Different types of sugar unites were found in fungal EPSs such as glucose, mannose, galactose, xylose, fucose, and rhamnose. It was also noticed that EPSs composed of the same monosaccharide units that were synthesized by different fungi had different molecular weight. This is caused by differing chain length or branching patterns that give polysaccharides a great diversity of structure, property, and functions. For instance, the extracellular polysaccharide produced by the mangrove-associated fungus Fusarium oxysporum Dzf17 is defined as galactofuranose-containing mannoglucogalactan, consisted of galactose, glucose, and mannose in a molar ratio of 1.33:1.33:1.00, and its molecular weight was about 61.2 $\mathrm{kDa}$ (Abdel-Azeem et al. 2019b).

\section{Mycotoxins and mycotoxicoses}

Mycotoxins are produced as toxic fungal secondary metabolites of different types of fungus, of enormous chemical diversity which belong primarily to genera Aspergillus, Penicillium and Fusarium. Mycotoxins play a significant role in food and feed safety, as well as in medical and environmental microbiology. They enter the food chain through contaminated crops, mainly cereals, which become infested prior to and during harvest, or during (improper) storage. They usually activated by signals from the environment, under favorable environmental conditions, when temperature and moisture are suitable, fungi proliferate and may synthesize toxins. Toxigenic molds are known to produce one or more secondary metabolites. Several hundreds of fungi types have been identified and reported; only a few are of practical significance causing toxicity to vertebrates and other animal groups in low concentrations. Most 
of the published data has concerned the major mycotoxins aflatoxins (AF), ochratoxins (OT), patulin, zearalenone (ZEA), and fumonisins (FUM) and trichothecenes (TCTs), especially deoxynivalenol (DON) and T-2/HT-2 toxin, produced from Aspergillus flavus, Aspergillus parasiticus, Fusarium graminearum, Fusarium culmorum, and Fusarium roseum (De Saeger et al. 2016; Ksenija 2018).

Mycotoxicoses is poisoning by natural means, and thus are analogous to the pathologies caused by exposure to pesticides or heavy metal residues. The symptoms of a mycotoxicosis depend on the type of mycotoxin, the amount and duration of the exposure, the age, health, and sex of the exposed individual; and many poorly understood synergistic effects involving genetics, dietary status, and interactions with other toxic insults. Thus, the severity of mycotoxin poisoning can be compounded by factors such as vitamin deficiency, caloric deprivation, alcohol abuse, and infectious disease status. In turn, mycotoxicoses can heighten vulnerability to microbial diseases, worsen the effects of malnutrition, and interact synergistically with other toxins (Bennett et al. 2013).

In the next decades, industry will have to face a new "mycotoxins scenario": the so-called "masked/bounded forms". In fact, many factors (e.g., heat, pressure, pH, enzymatic activities, and food constituents) must be considered during processing, since the release of native forms as well as formation of conjugated ones by reactions with macromolecular components (such as sugars, proteins or lipids) may be induced. Therefore, nowadays, the food industry clearly demonstrates the need for both rapid screening techniques, which could be also used outside the laboratory environment, and high sensitivity-precision methods for confirmatory purposes. Taking into consideration that, approximately $25 \%$ of the crops are contaminated by molds: the incidence can vary considerably depending on many factors, such as weather conditions, agricultural practices, packaging, transport and storage. Results show that almost $100 \%$ of these crops are contaminated with one or more mycotoxins (De Saeger et al. 2016).

What makes it more complicated the co-occurring mycotoxins. Regulations throughout the world do not consider the combined effects of mycotoxins. Several surveys have reported the natural co-occurrence of mycotoxins from all over the world concerning cereals and derived cereal product samples, among the 127 mycotoxin combinations described in the literature, AFs+FUM, DON+ZEA, AFs+OTA, and FUM+ZEA are the most observed. Studies of mycotoxin combination toxicity showed antagonist, additive or synergic effects depending on the tested species, cell model or mixture, and were not necessarily time- or dose-dependent (Smith et al. 2016).

\section{Mechanisms of mycotoxin-induced toxicity}

Reported mycotoxin disorders in digestive, immune, urinary and reproduction systems, have been in vivo and in vitro studied in order to clarify the mechanisms of mycotoxin-induced toxicity in these systems. In many cases, incorporation of mycotoxins into membrane structures causes various detrimental changes, resulting in alterations in second messenger systems through damaging membrane receptors. In addition, detrimental effects of mycotoxins on DNA, RNA and protein synthesis together with pro-apoptotic action further compromise important metabolic pathways and consequently changes in physiological functions including growth, development and reproduction occur. During the last decades, the importance of oxidative stress and lipid peroxidation was highlighted (Doi et al. 2011). As biochemical changes in mycotoxicosis vary greatly, the lipid peroxidation is regarded as one of the most important consequences of mycotoxicosis by generation of free radicals and subsequent oxidative stress causing structural and functional changes in the main organs for the metabolism of mycotoxins, liver and kidney. Moreover, the multiple mycotoxins contaminated diet may cause more serious damage than the single or pure mycotoxin (Rizzo et al. 1994; Hou et al. 2013). 
Oxidative stress refers to the in vivo oxidation and anti-oxidation imbalance reflects an imbalance between the systemic manifestation of reactive oxygen species and a biological system's ability to readily detoxify the reactive intermediates or to repair the resulting damage. Free radicals such as reactive oxygen species (ROS) are highly reactive, short-lived, toxic molecules that have one or more unpaired electrons and can damage DNA, proteins, lipids, and carbohydrates within the tissue, leading to many common diseases like atherosclerosis, cancer, and many others. In tissues, the primary antioxidant enzymes responsible for protection from oxidative stress by detoxifying free radicals are superoxide dismutase (SOD), catalase (CAT), and glutathione peroxidase (GPx). Mycotoxins are reported to decrease these antioxidant enzymes leading to tissue toxicity and carcinogenicity, and the main organs for their metabolism are liver and kidney as their antioxidant activity can be changed when feeding with overdoses of mycotoxins (Hou et al. 2013).

Antioxidants are the molecules, which prevent cellular damage by reducing the oxidative stress and therefore have a beneficial effect on human health could result in liver damage (Yilmaz et al. 2018). Delicate balance between antioxidants and pro-oxidants in the body in general and specifically in the cell is responsible for regulation of various metabolic pathways leading to maintenance of immune-competence, growth and development and protection against stress conditions. This balance can be regulated by dietary antioxidants (Surai et al. 2002).

\section{Strategies of mycotoxins prevention and decontamination}

Strategies to prevent, eliminate or inactivate mycotoxins in food and feed are urgently needed. Basic criteria were suggested when a decontamination strategy is assessed; (i) the mycotoxin must be inactivated by transformation to non-toxic com- pounds, (ii) fungal spores and mycelia should be destroyed, so that new toxins are not produced, (iii) the food or feed material should retain its nutritive value and remain palatable for consumers, (iv) the physical properties of raw material should not change significantly; it must be economically feasible (Santini et al. 2013).

Harmful effects of mycotoxin-contaminated food can be avoided by main four possibilities; preventing contamination, removing contaminated material from the food commodity, mitigating mycotoxin content in food by inhibition of absorption of mycotoxin in consumed food in the digestive tract, and treating exposed individuals (Medeiros et al. 2012). Plant disease management and adequate storage conditions, the good agricultural practices to limit mycotoxin levels, yet did not succeed to eliminate mycotoxins completely. Food processing can further reduce mycotoxin levels by physical removal, chemical or enzymatic transformation of mycotoxins into less toxic products. Physical removal of mycotoxins such as, manual sorting by farmers or automatic sorting by the industry significantly lowers the mean mycotoxin content. Chemicals transform mycotoxins into other compounds; must be assessed as the treatment does not impair nutritional quality, texture, or flavor of food. Recently defined criteria for detoxification techniques approval for feeds may serve as a model for the development of corresponding regulations for food (European Commission 2015). Further processing such as milling, steeping, and extrusion can also reduce mycotoxin content as mycotoxins can be detoxified chemically by reacting with food components and technical facilitated aids such as high temperature and alkaline or acidic conditions (Karlovsky et al. 2016).

Detoxification of mycotoxins can also be achieved enzymatically. Some enzymes naturally occur in food or which produced during fermentation are able to transform mycotoxins, but purified enzymes achieve more efficient detoxification. Integrating evaluation of processing technologies is recommended for its impact on mycotoxins into risk management. Processing steps proven to mitigate mycotoxin contamination should be used whenever necessary. Development of detoxification technologies for high-risk commodities should be a priority for research. While Physical and chemical degradation methods currently offer the most efficient 
post-harvest reduction of mycotoxin content in food, but they have some limitations such as limited efficacy, mycotoxin resistance to thermal treatment, safety issues, and losses in the nutritional value, as well as the expensive equipment required to implement these techniques (Leslie et al. 2014; Zadeike et al. 2018).

Biotransformation is a process by which organic compounds are transformed from one form to another to reduce the persistence and toxicity of the chemical compounds. This process is aided by major range of microorganisms and their products such as bacteria, fungi and enzymes (Singh 2017). The biotransformation process provides many advantages over chemical synthesis, as it can be carried out under mild conditions like ambient temperature and without the need of high pressure and extreme conditions reducing undesired byproduct, energy, and cost. The region-and stereo-selectivity of the process allows the production of enantiomerically pure compounds, eliminating the need for complicated separation and purification steps. Besides, the reactions occur under ecologically acceptable conditions, with lower emission of industrial resides and production of biodegradable resides and products, thus reducing the environmental problems. The products obtained by biotransformation process can be labeled as "natural". Comparing with chemical synthesis, that result in environmentally unfriendly production processes, possible formation of undesirable reaction mixtures, reduced process efficiency and increased downstream cost (Pimentel et al. 2011).

Biotechnology possesses the largest potential for future developments. Biological degradation of mycotoxins has shown to be promised because it works under mild, environmentally friendly conditions. Active metabolites isolated from microbial systems can convert mycotoxins with varied efficiency to non- or less toxic products. Fungi are remarkably a diverse group including approximately 1.5 million species, which can potentially produce a diverse array of secondary metabolites such as alkaloids, benzoquinones, flavanoids, phenols, steroids, tetralones, terpenoids, xanthones and anthraquinones, which have a tremendous impact on society and are exploited as antioxidants serve as the defensive factor against free radicals in the body. Researchers focused to screen and expand the spectrum of fungi having antioxidant potentials and to optimize the culture conditions to enhance their activities in pharmaceutical applications, in addition to safety considerations (Ji et al. 2016).

\section{Endophytic fungi as a source of dietary antioxidants}

Antioxidants, natural or synthetic food preservatives, are additives that preserve food and militate against oxidative deterioration on storage and processing. Due to their high stability and low volatility, the antioxidants help to maintain the level of nutrients, the texture, color, taste, freshness, functionality, aroma, and appeal to consumers. Antioxidants are essential for animal and plant life as well since they are involved in complex metabolic and signaling mechanisms (Wilson et al. 2017).

The term "endophyte" was first coined by Anton de Bary in the mid-19th century, then translated by Stergiopoulos and Gordon as "any fungus or bacterium found inside plant tissues" and by Rodriguez et al. (2009) as "the living together of dissimilar organisms". The most used definition includes also the life cycle: endophytes are endosymbiotic group of microorganisms that colonize plant tissues for a considerable part of their life cycle without causing disease to their host (Terhonen et al. 2019). Thus, the host itself has naturally served as a selection system for fungal species having bioactive molecules with reduced toxicity toward higher organisms of eukaryotic system. Endophytes are now considered as an important component of biodiversity. In order to protect their host from infectious agents and adverse conditions, they act as reservoirs of novel bioactive secondary metabolites, such as alkaloids, flavanoids, phenols, phenolic acids, quinones, steroids, saponins, tannins, terpenoids, isocoumarin derivatives, peptides and enzymes, beside their ability to produce the same or similar kind of compounds of from their originated host plants ( Padhi et al. 2013; Gouda et al. 2016). Many bioactive potential of endophytes have 
been proven, that served to nominate them as a potential candidate as antiviral, anticancer, antidiabetic, antimicrobial immunomodulatory, antitubercular, insecticidal and antiparasitic activities, and antioxidant capacity. In addition to the use of endophytic fungi in the stereoselective kinetic biotransformation of "thioridazine (THD)", phenothiazine neuroleptic drugs as these microorganisms are able to biomimic mammalian metabolism via biotransformation reactions. Another endophytic fungus was employed on the biotransformation of "propranolol (Prop)" to obtain 4-OH-Prop active metabolite in enantiomerically pure form (Singh 2017). While plant sources are being extensively explored for new chemical entities for therapeutic purposes, endophytic microbes also constitute an important source for drug discovery. In recent year's endophytic fungi attracting a lot of attention for their beneficial health- and growth-promoting effects for its ability to produce good number of new and interesting bioactive secondary metabolites, which are of pharmaceutical, industrial and agricultural importance ( Gunasekaran et al. 2017; Terhonen et al. 2019).

As a part of ongoing efforts towards finding novel antioxidant agents from natural resources, extracts of Endophytic fungi from all plants, either medicinal, rainforest or halophyte plants have attracted more attention as potential antioxidant resources in medicine, agriculture and industries (Padhi et al. 2013). Methods to obtain bioactive compounds include the extraction from a plant sources presents some disadvantages such as dependency on seasonal, climatic and political features and possible ecological problems in addition to increased food competition globally as consuming large amounts of edible materials. Thus calling for innovative approaches to obtain such compounds using different microorganisms appear promising alternatives for establishing an inexhaustible, cost-effective and renewable resource of high-value bioactive products and aroma compounds (Pimentel et al. 2011).

\section{Framework for hazard assessment of mycotoxins and regulations}

Recent global, regional and national food-borne disease outbreaks and/or large food recalls have had adverse impacts on consumer confidence in the safety of food supply and agrifood production and trade. Post-event analysis of such events has indicated the importance of more effective use of risk communication principles and practices.

(FAO/WHO 2016) stated a frame work for food hazard assessment that has been adopted by many national and regional food safety authorities around the world. The frame work is conducted through three main factors that work all together, risk assessment which defined as the process that is used to quantitatively or qualitatively estimate and characterize risk, risk management is the weighing and selecting of options and implementing controls as appropriate to assure an appropriate level of protection, and risk communication is defined as the exchange of information and opinions between people, specifically about risk and risk-related factors associated with food safety hazards (Fig. 2).

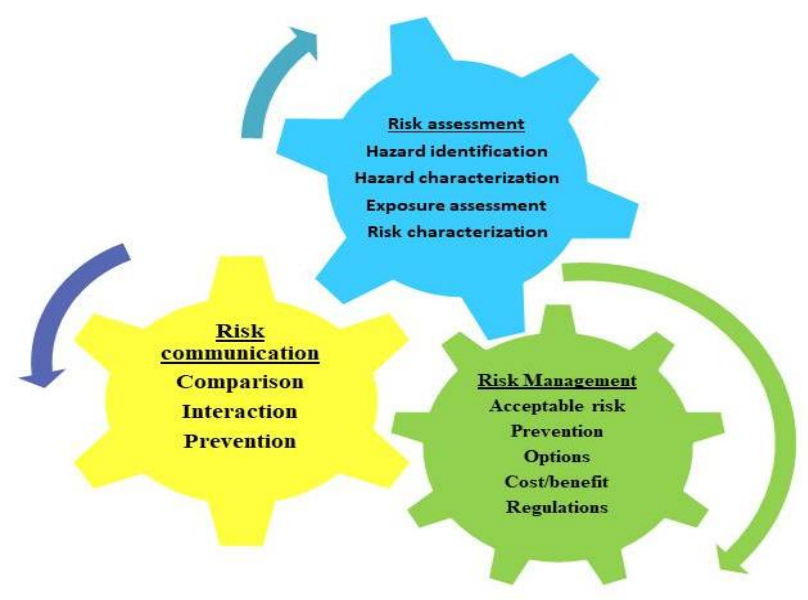

Fig. 2 - Framework of food hazard assessment. 
Mycotoxins presence in food is considered a real and severe risk to consumers for their toxicity. The challenges presented in mycotoxin research are enormous due to the frequency, the complexity and variability in occurrence. Several aspects make the pre- and post-harvest control of mycotoxins difficult, such as: Different fungal species produce mycotoxins, most of the mycotoxin producing fungi are able to produce more than one mycotoxin, mycotoxin levels are influenced by environmental conditions during growth and storage, the presence of modified mycotoxins and the highly complex influence of environmental factors on the biosynthesis of mycotoxins by fungi (De Saeger et al. 2017). Other aspects related to human and animal health also contribute to the complexity in mycotoxin research, e.g.; the lack of suitable biomarkers to assess exposure of humans and animals, the need for guidance levels of mycotoxins in animal body fluids, the efficacy and safety testing of mycotoxin detoxifiers, knowledge about toxicokinetics in men and animals and exposure is not the same due to different levels of contamination and dietary habits in various parts of the world (Vidal et al. 2018).

In human risk assessment, ingestion of food is considered a major route of exposure to mycotoxins. Oral bioavailability, defined as the fraction of an orally ingested mycotoxin that reaches the systemic circulation and is distributed throughout the body to exert its toxic effects, can be seen as the resultant of three processes: (i) release of the mycotoxin from its matrix into digestive juice in the gastrointestinal tract (bioaccessibility); (ii) transport of the mycotoxin across the intestinal epithelium into the vena Portae (intestinal transport); and (iii) biotransformation of the mycotoxin in the liver (and intestine) (metabolism) (Assunção et al. 2016). Despite of the practical difficulties in measuring the distribution and bioactivity of mycotoxins on a specific human body organ, the concept of bioaccessibility of mycotoxins has become important in the risk assessment considering that the amount of mycotoxin consumed via food (external dose) does not always reflect the amount available to the body (internal dose) to produce its toxic effects on target cells, tissues or organs (Vidal et al. 2018).

The need for regulations imposing limits to the concentration of mycotoxins in foods and feeds are generally recognized in developed countries and most of them have specific mycotoxin regulations as shown in map (Fig. 3), that illustrates countries with and without regulations for mycotoxins since 2004 (FAO) Food and Agriculture Organization of the United Nations 2004). Several factors, both of a scientific and socio-economic nature, may influence the establishment of mycotoxin limits and regulations depend on availability of toxicological data, data on the occurrence of mycotoxins in various commodities, availability of methods of sampling and analysis, implications for inter-country trade and the existence of sufficient food supply.

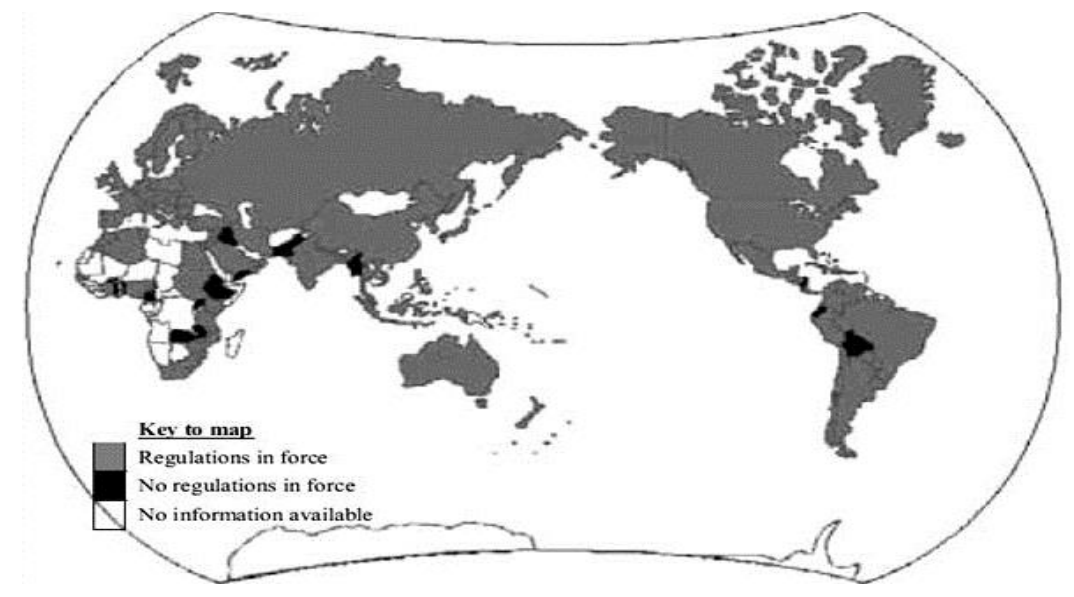

Fig. 3 - Countries with and without regulations for mycotoxins (FAO) Food and Agriculture Organization of the United Nations 2004). 
Most countries responded to this threat by establishing and enforcing maximum levels for mycotoxins in food (Commission of European Communities 2006a). Setting maximum levels is based on toxicity assessment and exposure data but it also takes supply and demand into account. Raw materials are usually tolerated to have higher contamination levels than finished products. The rationale behind this is either a dilution effect while preparation of the final product or the mitigation effects due to processing that lead to lowered mycotoxin concentration in the finished product than in the raw material (Karlovsky et al. 2016).

WHO in collaboration with FAO, assessed the risks to humans of mycotoxins through contamination in food and for recommending adequate protection. Risk assessments of mycotoxins in food were done by the Joint FAO/WHO Expert Committee on Food Additives (JECFA) which used by governments and by the Codex Alimentarius Commission (the intergovernmental standards-setting body for food) to establish maximum levels in food or provide other risk management advice to control or prevent contamination (Codex Alimentarius 2018).

The European Union (EU) introduced measures to minimize the presence of aflatoxins in different foodstuffs. Maximum levels of aflatoxins are laid down in (Commission of European Communities 2006b) Commission Regulation (EC) No. 1881/2006. For instance, in an opinion adopted in January 2007, the European Food Safety Authority (EFSA) scientific Panel on contaminants in the food chain (CONTAM), concluded that increasing the current EU maximum levels of $4 \mu \mathrm{g} / \mathrm{kg}$ total aflatoxins in nuts to 8 or $10 \mu \mathrm{g} / \mathrm{kg}$ total afatoxins would have had minor effects on the estimated dietary exposure, cancer risk and calculated margin of exposure. The Panel also concluded that exposure to aflatoxins from all food sources should be kept as low as reasonably achievable because aflatoxins are genotoxic and carcinogenic (Santini et al. 2013).

Furthermore, the presence of modified mycotoxins in food and feed has raised concern that modified mycotoxins may pose a non-negligible additional risk to human and animal health. Consequently, national and international agencies, including the European Food Safety Authority (EFSA) and the German Federal Institute for Risk Assessment (BFR), have launched efforts to address this emerging issue in food safety by developing strategies of critical assessment of potential added health risks of modified mycotoxins in food safety (Lorenz et al. 2019).

\section{Conclusion}

In today's changing world, safety and security have remained basic human needs, and securing safe food has been a major focus of international government action over the last years. Thus, Sustainable Development Goals (SDGs) 2030 target zero hunger, good health and wellbeing. Of both microbiological and chemical hazards, mycotoxins currently form a major food safety concern, for having serious effects on man and animals, which have led many countries to establish regulations on mycotoxins in food and feed to safeguard the health of humans and the food chain. The setting of mycotoxin regulations is a complex activity, which involves many factors and parties since the first limits for mycotoxins were lunched in the late 1960s for the aflatoxins. The need for new and useful compounds to provide assistance and relief in all aspects of the human condition is ever growing. Added to this are enormous difficulties in raising enough food on certain areas to support local human populations. Fungal endophytes constitute a major part of the unexplored fungal diversity, their metabolites represent the other side of the coin that could be employed in the deactivation of mycotoxins in food and feed as illustrated in Fig. (4). Therefore, there is a great necessity for further studies evaluating, managing and communicating the issue of mycotoxins of significant economic importance, with the goal of elucidating the public health risks. 


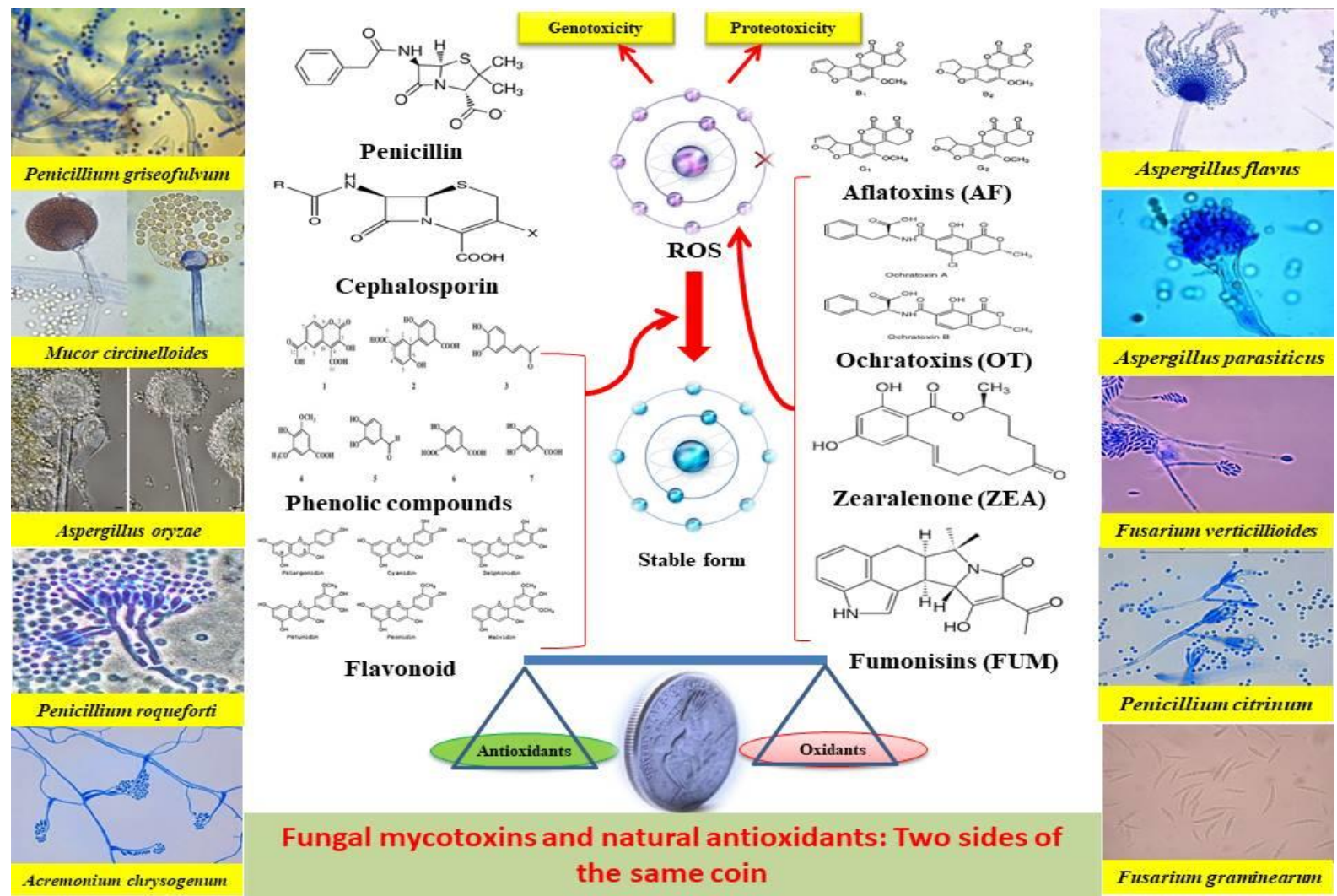

Figure 4 - Role of fungal antioxidants in deactivation of mycotoxins avoiding oxidative stress

\section{References}

Abdel-Azeem A.M., Abdel-Azeem M.A., Abdul-Hadi S.Y. and Darwish A.G. 2019a. Aspergillus: Biodiversity, Ecological Significances, and Industrial Applications. In Recent Advancement in White Biotechnology Through Fungi. Volume 1: Diversity and Enzymes Perspectives, 121-179. Switzerland AG.: Springer Nature.

Abdel-Azeem A.M., Abdel-Azeem M.A., Darwish A.G., Nafady N.A. and Ibrahim N.A. 2019b. Fusarium: Biodiversity, Ecological Significances, and Industrial Applications. In Recent Advancement in White Biotechnology Through Fungi. Volume 1: Diversity and Enzymes Perspectives, 201-261. Switzerland AG.: Springer Nature.

Abdel-Azeem A.M., Gherbawy Y.A. and Sabry A.M. 2016. Enzyme profiles and genotyping of Chaetomium globosum isolates from various substrates. Plant Biosystems, 150(3): 420-428.

Alnaimat S., Alharbi N.S., Alharbi S.A., Salmen S. H., Chinnathambi A., Al-Johny B.O. and Wainwright M. 2015. Mycelium of fungi isolated from mouldy foods inhibits Staphylococcus aureus including MRSA - A rationale for the re-introduction of mycotherapy. Saudi Journal of Biological Sciences, 22(5): 600-603. Available at: http://dx.doi.org/10.1016/j.sjbs.2015.06.013.

Assunção R., Silva M.J. and Alvito P. 2016. Challenges in risk assessment of multiple mycotoxins in food. World Mycotoxin Journal, 9(5): 791-811.

Bennett J.W. and Klich M. 2013. Micotoxins. Clinical microbiology reviews 16(3): p.497-516. Available at: https://www.ncbi.nlm.nih.gov/pmc/articles/PMC164220/.

Chahal D.S. and Hawksworth D.L. 1976. Chaetomium cellulolyticum, a new thermotolerent and cellulolytic Chaetomium. I. Isolation, description and growth rate. Mycologia, 68(3): 600- 
610.

Codex Alimentarius Commission 2018. General standard for contaminants and toxins in food and feed, CXS 193-1995. Available at: https://www.uam.es/gruposinv/meva/publicaciones jesus/capitulos_espanyol_jesus/2005_motivacion para el aprendizaje Perspectiva alumnos.pdf\%0Ahttps://www.researchgate.net/profile/Juan_Aparicio7/publication/2535713 79_Los_estudios_sobre_el_cambio_conceptual_y_.

Commission of European Communities 2006a. Communication from the commission to the European Parliment and the Council, on action for safer Europe.

Commission of the European Communities 2006b. Commission Regulations EC 1881/2006. Setting maximum levels for certain contaminants in foodstuffs. Official Journal of the European Union, 364(1881): 5-24.

Doi K. and Uetsuka K. 2011. Mechanisms of Mycotoxin-Induced Neurotoxicity through Oxidative Stress - Associated Pathways. International Journal of Molecular Sciences, 12: 5213-5237.

EFSA. 2018. EFSA - European Food Safety Authority Panel on Contaminants in the Food Chain (CONTAM), Risk to human and animal health related to the presence of 4,15diacetoxyscirpenol in food and feed. EFSA Journal, 16(8).

Eriksen J. and Goksoyr J. 1977. Cellulases from Chaetomium thermophile var. dissitum. Europian Journal of Biochemistry, 77: 445-450.

European Commission 2015. Commission Regulations EC 2015/786 defining accept- ability criteria for detoxification processes applied to products intended for animal feed. Official Journal of the European Union, 125: 10-14.

FAO, Food and Agriculture Organization of the United Nations. 2004. Worldwide regulations for mycotoxins in food and feed in 2003. FAO Food and Nutrition Paper, 81: 9-28. Available at: ftp://ftp.fao.org/ (accessed on 15 April 2019).

FAO/WHO. 2016. A handbook on risk communication applied of food safety. World Health Organization Food Agriculture Organization of the United Nations.

Gillot G., Jany J., Coton M., Le Floch G., Debaets S., Ropars J., López-Villavicencio M., Dupont J., Branca A., Giraud T. and Coton E. 2015. Insights into Penicillium roqueforti morphological and genetic diversity. PLoS ONE, 10(6): 1-21.

Gouda S., Das G., Sen S.K., Shin, H.S. and Patra J.K. 2016. Endophytes: A treasure house of bioactive compounds of medicinal importance. Frontiers in Microbiology 7(SEP): 1-8.

Green B.J. and Beezhold D.H. 2011. Industrial Fungal Enzymes: An Occupational Allergen Perspective. Journal of Allergy, 2011: 1-11.

Gunasekaran S., Sathiavelu M. and Arunachalam S. 2017. In vitro antioxidant and antibacterial activity of endophytic fungi isolated from Mussaenda luteola. Journal of Applied Pharmaceutical Science, 7(8): 234-238.

Hou Y.J., Zhao Y.Y., Xiong B., Cui X.S., Kim N.H., Xu Y.X. and Sun S.C. 2013. MycotoxinContaining Diet Causes Oxidative Stress in the Mouse. PLoS ONE, 8(3): 2-8.

Hymery N., Vasseur V., Coton M., Mounier J., Jany J.L., Barbier G. and Coton E. 2014. Filamentous fungi and mycotoxins in Cheese: A review. Comprehensive Reviews in Food Science and Food Safety, 13(4): 437-456.

Ianiro G., Pecere S., Giorgio V., Gasbarrini A. and Cammarota G. 2016. Digestive Enzyme Supplementation in Gastrointestinal Diseases. Current Drug Metabolism, 17(2): 187-193.

Ji C., Fan Y. and Zhao L. 2016. Review on biological degradation of mycotoxins. Animal Nutrition, 2(3): 127-133. Available at: http://dx.doi.org/10.1016/j.aninu.2016.07.003.

Karlovsky P., Suman M., Berthiller F., De Meester J., Eisenbrand G., Perrin I., Oswald I.P., Speijers G., Chiodini A., Recker T. and Dussort P. 2016. Impact of food processing and detoxification treatments on mycotoxin contamination. Mycotoxin Research, 32(4): 179205. Available at: http://dx.doi.org/10.1007/s12550-016-0257-7. 
Ksenija N. 2018. Mycotoxins - climate impact and steps to prevention based on prediction. Acta Veterinaria, 68(1): 1-15.

Langdon S., Pearce C.J. and Ph D. 2017. The Microbial Pharmacy: FDA Approved Medicines From Fungi. Mycosynthetix, 18: 1-4.

Leslie J.F. and Logrieco A.F. 2014. Mycotoxin Reduction in Grain Chains. Wiley Blackwell.

Lessard M.H., Viel C., Boyle B., St-Gelais D. and Labrie S. Metatranscriptome analysis of fungal strains Penicillium camemberti and Geotrichum candidum reveal cheese matrix breakdown and potential development of sensory properties of ripened Camembert-type cheese. BMC Genomics, 15(1): 1-13. Available at: BMC Genomics.

Lorenz N., Dänicke S., Edler L., Gottschalk C., Lassek E., Marko D., Rychlik M. and Mally A. 2019. A critical evaluation of health risk assessment of modified mycotoxins with a special focus on zearalenone. Mycotoxin Research 35: 27-46. doi.org/10.1007/s12550-018-0328-z

Mandell G.L., Douglas J.R., Gordon R. and Bennett J.E. 1992. Principles and practice of infectious diseases: handbook of antimicrobial therapy. New York: Churchill Livingstone.

Medeiros F.H.V., Martins S.J., Zucchi T.D., Melo I.S., Batista L.R. and Machado J.C. 2012. Biological control of mycotoxin-producing molds. Ciência e Agrotecnologia, 36(5): 483497.

Mehta A., Bodh U. and Gupta R. 2017. Fungal lipases: A review. Journal of Biotech Research, 8(1): 58-77.

Muñiz C.C., Zelaya T.E.C., Esquivel G.R. and Fernández Perrino F.J. 2007. Penicillin and cephalosporin production: A historical perspective. Revista Latinoamericana de Microbiologia, 49(3-4): 88-98.

Padhi L., Mohanta Y.K. and Panda K.S. 2013. Endophytic fungi with great promises : A Review. Journal of Advanced Pharmacy Education \& Research, 3(3):152-170.

Penesyan A., Gillings M. and Paulsen I.T. 2015. Antibiotic discovery: Combatting bacterial resistance in cells and in biofilm communities. Molecules, 20(4): 5286-5298.

Pimentel M.R., Molina G., Dion A.P. and Pastore M. 2011. The use of endophytes to obtain bioactive compounds and their application in biotransformation process. Biotechnology Research International, 2011:1-11.

Pitt J.I., and Hocking A.A.D. 2009. Fungi and food spoilage 3rd ed. New-York: Springer. Richard J.L. 2008. Discovery of aflatoxins and significant historical features. Toxin Reviews, 27: 171-201.

Rizzo A.F., Atroshi F., Ahotupa M., Sankari S. and Elovaara E. 1994. Protective effect of antioxidants against free radical-mediated lipid peroxidation induced by DON or T-2 toxin. Journal of Veterinary Medicine Series A, 41: 81-90.

Rodriguez R.J., Jr J.F.W., Arnold A.E. and Redman R.S. 2009. Fungal endophytes : diversity and functional roles. New Phytologist, 182: 314-330.

De Saeger S., Audenaert K. and Croubels S. 2016. Report from the 5th International Symposium on Mycotoxins and Toxigenic Moulds: Challenges and Perspectives (MYTOX) Held in Ghent, Belgium, May 2016. Toxins, 8(5): 146.

De Saeger S., and Logrieco A. 2017. Report from the 1st MYCOKEY International Conference Global Mycotoxin Reduction in the Food and Feed Chain Held in Ghent, Belgium, 11-14 September 2017.

Santini A. and Ritieni A. 2013. Aflatoxins: Risk, Exposure and Remediation. In Aflatoxins Recent Advances and Future Prospects, 343-376. InTech

Singh R. 2017. Microbial Biotransformation: A Process for Chemical Alterations. Journal of Bacteriology \& Mycology: Open Access, 4(2): 47-51.

Smith M.C., Madec S., Coton E. and Hymery N. 2016. Natural Co-occurrence of mycotoxins in foods and feeds and their in vitro combined toxicological effects. Toxins, 8(94) doi:10.3390/toxins8040094. 
Souza P.M., Bittencourt M.L.A., Caprara C.C., Freitas M., Almeida R.P.C., Silveira D., Fonseca Y.M., Filho E.X.F., Junior A.P. and Magalhaes P.O. 2015. A biotechnology perspective of fungal proteases. Brazilian Journal of Microbiology 46(2): 337-346. doi: http://dx.doi.org/10.1590/S1517-838246220140359

Surai F. and Dvorska E. 2002. Impact of mycotoxins on the body's antioxidant defense. In Nutritional Biotechnology in the Feed and Food Industries, 131-141. Nottingham University Press Available at: http://en.engormix.com/MA-mycotoxins/articles/impactmycotoxins-bodys-antioxidant-t391/AVG-p0.htm.

Suseela L., Anupama M., Prudhvilal B., Narasaiah T.V. and Latha J.N.L. 2014. Isolation \& charecterization of lipase producing fungi from palm oil mill effluent obtained from Pedavegi, A.P., India. International Journal of Biological \& Pharmaceutical Research, 5(7): 559-565. Available at: www.ijbpr.com.

Terhonen E., Blumenstein K., Kovalchuk A. and Asiegbu F.O. 2019. Forest tree microbiomes and associated fungal endophytes: Functional roles and impact on forest health. Forests, 10(1):1-32.

Thom, Charles, Humfeld, Harry and Holman H.P. 1934. Laboratory tests for mildew resistance of outdoor cotton fabrics. American Dyestuff Reporter (ADR), Illus. 23(581): p.586.

Treseder K.K. and Lennon J.T. 2015. Fungal traits that drive ecosystem dynamics on land. Microbiology and Molecular Biology Reviews, 79(2): 243-262. Available at: http://mmbr.asm.org/lookup/doi/10.1128/MMBR.00001-15.

Vidal A., Mengelers M., Yang S., De Saeger S. and De Boevre M. 2018. Mycotoxin biomarkers of exposure: A comprehensive review. Comprehensive Reviews in Food Science and Food Safety, 17(5): 1127-1155.

Wilson D., Nash P., Buttar H., Griffiths K., Singh R., De Meester F. and Horiuchi R. 2017. The role of food antioxidants, benefits of functional foods, and influence of feeding habits on the health of the older person: An Overview. Antioxidants, 6(4): 81. Available at: http://www.mdpi.com/2076-3921/6/4/81.

Yadav L.S. and Bagool R.G. 2015. Isolation and screening of cellulolytic Chaetomium sp. from deteriorated paper samples. International Journal of Current Microbiology and Applied Science, 4(8): 629-635.

Yilmaz S., Kaya E. and Kisacam M.A. 2018. The effect on oxidative stress of aflatoxin and protective effect of lycopene on aflatoxin damage. In Aflatoxin-Control, Analysis, Detection and Health Risks, 13. Book Citation Index in Web of Science ${ }^{\mathrm{TM}}$ Core Collection (BKCI)

Zadeike D., Juodeikiene G. and Bartkiene E. 2018. Recent strategies for microbial and fungal decontamination of cereals with a potential for application in food production. Journal of Biotechnology and Bioengineering, 2(3): 13-18. 